\title{
Oeffentliches Sanitätswesen.
}

\section{Aus dem preussischen Medizinaletat für das Jahr 1901.}

Dauernde Ansgaben. Universitätsinstitute. Zur Errichtung eines Extraordinariats für gerichtliche Medizin in Königsberg werden 3410 M. gefordert. - Berlin. Die neu eingerichteten Unterrichtsstellen für Mechanotherapie und für Massage erfordern einen Aufwand von zusammen 9200 M.; davon entfällt auf den Lehrer der Massage eine Remuneration von 1800 M. Die Subvention für den Unterricht in der Mechanotherapie beträgt 5000 M.; zweifellos kommt dem Ministerium die Subvention eines privaten Instituts billiger, als wenn es eine eigene Anstalt bauen und unterhalten müsste. Eine "lobenswerthe "fiskalische Sparsamkeit! Immerhin wird derselbe Zweck in Breslau (s. unter „Einmalige Ausgaben") noch billiger und - wie es scheint - zweckmässiger auf anderem Wege erreicht. - Für den neuen Unterricht in der Hydrotherapie an der Charité sind $4300 \mathrm{M}$. eingestellt. - In Greifswald ist die Umwandlung des Extraordinariats für Psychiatrie in ein Ordinariat geplant; die durch den Tod Arndt's erledigte Stelle ist einstweilen noch nicht besetzt. - Auch in Breslau soll ein Extraordinariat für gerichtliche Medizin eingerichtet werden. Ebenso ein neues Extraordinariat für innere Medizin (ausnahmsweise mal) mit Gehalt (3410 M.). - - In Halle wird ein (künftig wegfallendes) Ersatzordinariat errichtet, ferner ein Extraordinariat für gerichtliche Medizin. - Für die neue Universitätsirrenklinik $\mathrm{K}$ iel's, zu deren Leitung be- 
kanntlich Prof. Siemerling berufen ist, ist eine grössere Summe ausgeworfen. - In Göttingen wird ein (künftig wegfallendes) Ordinariat errichtet. - In Bơn wird ein Extraordinariat für Chirurgie errichtet, ebenso ein Extraordinariat für gerichtliche Medizin. - Erwähnung verdient schliesslich, dass beabsichtigt ist, die etatsmässige Remuneration von solchen Universitätsassistenten, welche sich bewährt haben und deren längeres Verbleiben in ihrer Stellung im Interesse des Instituts liegt, je nach Bedarf im Einzelfalle bis zum Höchstbetrage von 1800 M. zu erhöhen; zu dem Zweck ist ein Fonds von 8000 M. in den Etat eingestellt.

Medizinalwesen. In der Medizinalabtheilung des Ministeriums soll eine vierte Rathstelle (Anfangsbesoldung $7500 \mathrm{M}$.) geschaffen werden, da ,die sowohl nach ihrem äusseren Umfange wie nach ihrer inneren Bedeutung wachsenden Aufgaben auf allen Gebieten der Medizinalverwaltung eine dauernde Verstärkung der technischen Kräfte an der Centralstelle zur dringenden Nothwendigkeit machen." Also wieder ein hochbedeutsames Stück Medizinalreform! Hieran reiht sich die Einrichtung einer zweiten Regierungs- und Medizinalrathsstelle beim Berliner Polizeipräsidium (4200 M. Gehalt). „Bei dem Polizeipräsidium in Berlin ist neben dem Regierungs- und Medizinalrath ein Medizinalassessor beschäftigt. Letzterer wird in der Regel aus der Reihe derjenigen Kreismedizinalbeamten entn^mmen welche zur Beförderung zum Regierungs- und Medizinalrath empfohlen sind. Die bisherigen Inhaber dieser Stelle sind daher nur eine verhältnissmässig kurze Zeit in derselben gewesen. Um einen häufigen Wechsel zu vermeiden, und da die Thätigkeit des Medizinalassessors derjenigen eines Regierungs- und Medizinalraths gleichwerthig ist, erscheint die Umwandlung der Stelle in die eines Regierungs- und Medizinalraths erforderlich."

Ueber die Zahl der übrigen Medizinalräthe und der neu zu schaffenden Kreisarzt- und Kreisarztassistentenstellen vergl. den speziellen Artikel in No.3, S. 47. - Als Zuschuss für das Charitékrankenhaus sind $309253 \mathrm{M}$. eingestellt; darunter verdient die Summe von $900 \mathrm{M}$. zur Erliöhung der Besoldung des ersten Assistenten am Pathologischen Institut besondere Erwähnung. Beim Institut für Infektionskrankheiten erhält der Assistent, welcher Leiter des Laboratoriums für besonders gefährliche Krankheiten ist, eine Gehaltserhöhung von 650 M. (also in Summa jährlich 2000 M.). - Zur Unterhaltung der neu zu schaffenden staatlichen Versuchs- und Prüfungsanstalt für die Zwecke der Wasserversorgung und Abwässerbeseitigung ist ein Jahresbetrag von $45000 \mathrm{M}$. vorgesehen. Die Einrichtung des neuen Instituts wird folgendermaassen begründet. „Die hygienisch einwandfreie Wasserzuführung und Abwässerbeseitigung ist eine der wesentlichsten Vorbedingungen für eine günstige wirthschaftliche Entwickelung volkreicher Gemeinwesen und das vornehmste Mittel, dem Ausbruche von Krankheiten und ihrer epidemischen Ausbreitung vorzubeugen. Diese Bedeutung ist allseitig erkannt und neuerdings auch in einer dem Staatsministerium vorgelegten Petition von Vertretern grösserer Städte und Industrieen vom 24. März 1900 wegen Errichtung einer staatlichen Prüfungsanstalt für diese Zwecke hervorgehoben worden. In dieser wird zugleich auf die Schäden hingewiesen, welche den Gemeinden und Industrieen durch unzweckmässige Maassnahmen auf dem Gebiete der Trinkwasserversorgung und Abwässerbeseitigung entstanden sind, weil es an einer Stelle fehlt, wo die sich auf diesem Gebiete vollziehenden Vorgänge und die daraus zu ziehenden praktischen und wissenschaftlichen Erfahrungen einheitlich gesammelt und verwerthet werden. Die vielfach geschaffenen fehlerhaften Anlagen haben bedeutende wirthschaftliche Schäden und beklagenswerthe hygienische Missstände - namentlich Verunreinigungen der Flussläufe -... mit sich geführt. Diese Umstände haben die Aufsichtsbehörden veranlasst, seit Jahren die planmässige Forschung auf diesem Gebiete anzuregen und namentlich die Ergebnisse der verschiedenen Methoden für die Reinigung der Abwässer nachprüfen und Untersuchungen über die Nutzbarmachung der Abwässer und der Klärrückstände für die Landwirthschaft, sowie über die Selbstreinigung der Flüsse u. dergl. anstellen zu lassen. Die Angelegenheit ist von einer aus den Vertretern der betheiligten Ministerien zusammengesetzten Commission bisher mit Erfolg geführt worden. Gegenüber der stets wachsenden Bedeutung und dem Umfange der übernommenen. Aufgaben erweist sich die bisherige Organisation und die Heranziehung einzelner Sachverständiger von Fall zu Fall nicht als ausreichend. Es sollen daher die von jener Commission bisher veranlassten Arbeiten durch die versuchsweise Schaffung einer Centralstelle, bei welcher alle einschlagenden hygienischen und volkswirthschaftlichen Interessen Berücksichtigung und planmässige Förderung finden, gesichert werden. Das Institut soll zugleich den Staatsbehörden, Communen und Industrieen etc. die Möglichkeit für die Einholung von objektiver, sachkundiger, nach dem jeweiligen Stande von Wissenschaft und Praxis einzurichtender Auskunft gewähren. Die Anstalt wird der Medizinalabtheilung des Ministeriums der geistlichen etc. Angelegenheiten angegliedert, während die Aufsicht über dieselbe von einer Commission, bestehend aus den Vertretern der betheiligten Ministerien, ausgeübt wird. Die geschäftliche Leitung der Anstalt wird einem vortragenden Rathe der Medizinalabtheilung übertragen, welcher zugleich den Vorsitz in der Commission führt und die letztere nach aussen und der Anstalt gegenïber vertritt. Das wissenschaftliche Personal der Anstalt soll aus einem Vorsteher, dem die technische Leitung obliegt, einigen wissenschaftlichen Mitarbeitern und den zu einzelnen Zwecken hinzuzuziehenden Hilfsarbeitern bestehen, denen die nöthige Bureauhilfe und ein genügendes Unter personal zur Seite gestellt wird. Unter dem wissenschaftlichen Personal ist je ein Chemiker, ein bakteriologisch gebildeter Arzt, ein Botaniker und ein Ingenieur vorgesehen. Die Anstalt soll zunächst provisorisch eingerichtet werden, damit erst über ihre Wirksamkeit und ihren Geschäftsumfang Erfahrungen gesammelt werden können. Es ist ins Ange gefasst, die dauernden Ausgaben durch Erhebung von Gebühren für die von Behörden und Privaten beantragten Prüfungen, Begutachtungen, Ueberwachungen etc. nach Maassgabe eines aufzustellenden Tarifs thunlichst zu decken."

Für medizinal-polizeiliche Zwecke einschliesslich $15000 \mathrm{M}$ zur Bestreitung der Kosten der sanitätspolizeilichen Controlle behufs Abwehr der Choleragefahr und $15000 \mathrm{M}$. für das Leprakrankenheim im Kreise Memel sind $100000 \mathrm{M}$. ausgeworfen.

Einmalige und ausserordentliche Ausgaben. Alle diejenigen, welchen je das zweifelhafte Vergnügen zu Theil geworden ist, in einem Wartezimmer der Unterrichts- oder Medizinalabtheilung antichambriren $\mathrm{zu}$ müssen, werden mit grossem Interesse vernehmen, dass für das "Geschäftshaus" des Ministeriums ein Neubau geplant ist. Für den Ankauf eines benachbarten Grundstückes sind 854250 M., für den Neubau als erste Rate 600000 M. eingestellt. - In König-sberg werden zu "baulichen Herstellungen“ der chirurgischen Klinik 89500 M. gefordert. Der im vorigen Jahre bewilligte Bau einer Trachomkrankenbaracke giebt zll einer Nachforderung von 16000 M. Veranlassung. - In der Berliner Poliklinik für Haut und Geschlechtskrankheiten sollen Versuche mit eiektrischer Licht behandlung angestellt werden, wozu $30000 \mathrm{M}$. verlangt werden. Die Sammlıng von Wachsmodellen bei der Klinik für Haut- und Geschlechtskrankheiten - auf deren Werth für den Unterricht ich schon vor Jahren hinzuweisen Gelegenheit hatte - soll endlich vervollständigt werden (10000 M.). Zur Herrichtung und zur Ausstattung von Räımen für den Unterricht in der Massage sind $8000 \mathrm{M}$. angesetzt. - In Greifs wald soll die medizinische und chirurgische Klinik durch neue Grundstücke erweitert werden $(111150 \mathrm{M}$.). Zur Beschaffung von Instru menten und Apparaten für die medizinische Klinik sowie zur Vervoll ständigung der Bibliothek derselben sind $20000 \mathrm{M}$. erforderlich. In der Begriindung dieser Position heisst es, es habe sich gelegentlich eines Wechsels in der Leitung der Klinik ergeben, dass die Anstalt hinsichtlich ihrer Ausstattung mit Instrumenten etc. hinter den übrigen medi zinischen Kliniken zurückgeblieben sei und dass die obige Summe nöthig sei, um geordnete Verhältnisse herbeizuführen. (Derselbe schmeichelhafte „Epilog“ für den frïheren Leiter der Klinik findet sich bei der medizinischen Klinik in Halle a./S.) Als zweite Rate zum Neubau der chirurgischen Klinik werden 200000 M., zum Umund Erweiterungsbau der Franenklinik 88200 M. gefordert. - Zur Erweiterung der chirurgischen Klinik in Breslau (Schaffung von Räumen für Medikomechanik [cf. Berlin!] und Orthopädie etc.) sind 85000 M. zu bewilligen. Für Unterrichtsmittel der Klinik für Syphilis und Hautkrankheiten, sowie zu baulichen Herstellungen sind $21450 \mathrm{M}$ eingestellt. Die zweite Rate zum Neubau der Kinderklinik und -Poliklinik beträgt 88000 M. - Halle, Medizinische Klinik siehe oben. - In Kiel beträgt die erste Rate zur Erweiterung des Anatomischen Instituts 100000 M. Zur Vergrösserung des Grundstïcks der Akademischen Heilanstalten sind 124500 M. nöthig. (Auf die bekannte Affäre "Quincke" bezieht sich der in der Begründung ent haltene Satz: "nachdem sich dem Neubau der medizinischen Klinik auf dem dafür in Aussicht genommenen Theile des Geländes der Heilanstalten Schwierigkeiten entgegengestellt haben, wird beabsichtigt, einen Neuban für die chirurgische Klinik herzustellen. Die letzte Rate zum Neubau einer Universitäts-Irrenanstalt beträgt 458900 M. - In Göttingen "hat sich im Laufe des letzten Sommers helausgestellt, dass die Einrichtungen der medizinischen Klinik zur Absonderung von Kranken mit ansteckenden Krankheiten nicht genügen." Die Absonderungsbaracke soll deshalb erweitert werden (6000M.) - Marburg. Letzte Rate zum Neubau des Anatomischen Instituts 151000 M. Für das Hygienische Institut zur Anstellung von (,noch nicht abgeschlossenen ") Versuchen mit Tuberkulosegiftpräparaten gegen die Rindertuberkulose sind $20000 \mathrm{M}$. erforderlich. - In Bonn soll eine hydrotherapeutische Abtheilung bei der medizinischen Klinik (für 56000 M.) geschaffen werden. Zweite Rate zum Neubau der Augenklinik 120000 M. - - Schliesslich werden an Zuschüssen an Universitäts institute- für Untersuchungen mit Röntgenstrahlen 20000 M., zur Be schaffung von Instrumenten und Apparaten für subventionirte Polikliniken $16000 \mathrm{M}$. gefordert.

Auf dem Gebiete des Medizinalwesens sind folgende einmalige Positionen vorhanden: Ergänzungsrate zum Neubau des Instituts für Infektionskrankheiten 156000 M., zur Bekämpfung der Granulose 350000 M., zur Untersuchung der Maul- und Klauenseuche 30000 M. zur Bestreitung der Kosten der ersten Anschaffung von Apparaten etc. für die Kreisärzte, sowie zur Gewährung von Beihilfen an dieselben 
zur Beschaffung von Apparaten etc. 50000 M., für den Neubau der Charité weitere $1089630 \mathrm{M}$. J. S. 